\title{
ARTICLE
}

\section{Experience using models imported from CAD software for shielding calculations in MCBEND}

\author{
Adam Bird* and Albrecht Kyrieleis
}

\author{
AMEC, Kimmeridge House, Dorset Green Technology Park, Winfrith Newburgh, DT2 8ZB, United Kingdom
}

\begin{abstract}
Reactor components (e.g. fuel assemblies) and other equipment (e.g. transport flasks) are designed using Computer Aided Design (CAD) packages. The process of converting CAD models into models that can be used in a Monte Carlo simulation is both time consuming and a source of approximations and errors. It is useful to be able to directly use these geometries in Monte Carlo calculations. MCBEND Version 11A Release Update 0 includes several methods of importing CAD geometries. The first method provides full accurate support for the range of geometry capabilities offered by the Initial Graphics Exchange Specification (IGES) model which can be used in combination with the existing geometry capabilities of MCBEND. All the surfaces in the IGES model are used directly with no approximation. This capability is provided by the OiNC software package developed by Sellafield Ltd as part of a Nuclear Code Development collaboration between Sellafield Ltd and the ANSWERS Software Service. A further two methods provide high performance CAD import options, but with a geometry approximation introduced by first converting the CAD file to a tetrahedral mesh representation or polygon surface representation, known as Standard Tessellation Language (STL), before importing them into MCBEND.
\end{abstract}

\section{Keywords: MCBEND; geometry; CAD import; Monte Carlo; radiation transport code}

\section{Introduction}

This paper reports on exercising the CAD import capabilities of the ANSWERS Monte-Carlo radiation transport code MCBEND11A RU0 [1].

The discussion will focus on the IGES import capability since the tetrahedral mesh import has been previously reported [2].

The CAD import has been tested with a series of simple geometries designed to verify specific features of the CAD format. Apart from the Hexagonal Pipe and Human Body all the examples presented here are based on real world MCBEND calculations.

\section{CAD related developments for MCBEND11}

As reported in [2] the performance of the tetrahedral mesh geometry is independent of the number of meshes and is dependent instead on the memory use of the optimization function. With the release of MCBEND11 the memory requirements of the optimization function have been reduced. Any number of tetrahedral mesh models may be imported and these can be freely scaled, rotated, replicated and integrated with all the other geometry capabilities of MCBEND.

The IGES and the STL import capability are provided by the OiNC [3] software package developed by Sellafield Ltd as part of a Nuclear Code Development

*Corresponding author. Email: adam.bird@amec.com collaboration between Sellafield Ltd and the ANSWERS Software Service. A single IGES or STL model can be imported. This can be freely rotated, replicated and integrated with all the geometry capabilities of MCBEND.

The IGES file format is flexible such that many different representations (or entity types) can be used to define the same geometry. MCBEND has mainly been tested using IGES MSBO (Manifold Solid B-rep Objects) format files generated by SolidWorks and Autodesk Inventor, and IGES trimmed NURBS (Non Uniform Rational B-Splines) format from Autodesk Inventor. The IGES import capability has not yet been tested with IGES files from a wide range of CAD packages so the capability is being offered as an evaluation feature in MCBEND11. MCBEND models with imported CAD geometries can be visualized in Visual Workshop. Details of the CAD model, including assigned material names and numbers, are displayed. This enables verification that the model has been correctly imported.

\section{Use in MCBEND}

A tetrahedral mesh model, when imported, becomes a standard 'Hole' in the MCBEND geometry and can be used in the same way as other Holes.

\footnotetext{
a A 'Hole' in MCBEND geometry context is a volume of space that uses Woodcock tracking.
} 
An IGES or STL model is referenced in the MCBEND geometry section as a body of type 'IGES' or 'STL'. This body can be used in almost exactly the same way as all other MCBEND body types even though it is a collection of all the entities that make up the IGES or STL model. It can be placed in all types of parts; it can be freely rotated, shifted and replicated. A MCBEND body can be placed inside an IGES body. In this way the CAD model is integrated in a complex MCBEND geometry in an easy and flexible manner.

Volumes of space that contain physical materials within the CAD model are assigned MCBEND material names using a simple convention, for example 'IGES1, IGES2 etc. if no other directives are given in the CAD model. Volumes within the CAD model boundary but not defined can be assigned to an interstitial material. The compositions of these materials are defined in the standard MCBEND fashion.

\section{Example calculations}

Each model has been created in two ways; first as a Simple Body (known in MCBEND as Fractal Geometry or FG) / Hole geometry, and second as a (IGES) CAD model using the Solidworks software or a polygon surface model. The CAD versions are completely defined in CAD with MCBEND geometry being used to locate the model in space and provide scoring volumes. Track length scoring is used in all cases. The scoring volumes are identical between CAD and FG models. In all cases the geometry can be replicated exactly or very nearly exactly using the standard geometry capabilities of MCBEND. Variance reduction methods are identical between the CAD and FG versions. The Transport flask uses splitting and roulette utilising a radial exponential importance map. The Fuel flask uses splitting and roulette utilising a space energy importance map automatically calculated by MCBEND. No variance reduction was used for the other calculations. The standard deviation is less than $6 \%$ for most compared results, exceptions being some results from the Fuel and Transport flasks which are $15-20 \%$ for the CAD cases because of longer run times. As far as possible the calculations are identical apart from the method of defining the physical geometry. This allows meaningful comparison of the performance of particle tracking algorithms. The images are generated using Visual Workshop [4].

\subsection{Storage room}

A room with concrete walls and a duct is modelled containing steel barrels, Figure 1. Some of the barrels (blue) are filled with a uranium/concrete mixture and act as a gamma source, the other barrels are empty (yellow). The line spectrum of the gamma source includes the 1.33, 1,17 and $0.662 \mathrm{MeV}$ lines of ${ }^{137} \mathrm{Cs}$ and ${ }^{60} \mathrm{Co}$.

The fluxes have been scored in 5 regions just above a row of barrels (red line in Figure 1). The results from the $\mathrm{FG}$ and the CAD model agree within 1 standard deviation.

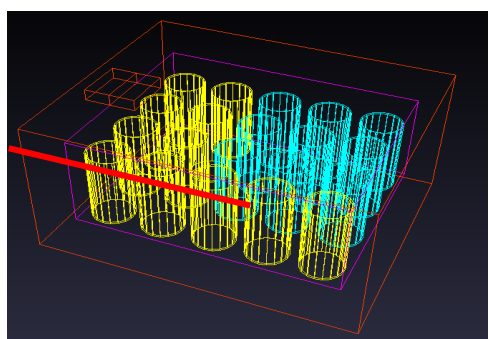

Figure 1. The storage room model. The red line marks the direction the flux profile has been obtained in.

\subsection{Transport flask}

A steel/lead transport container is modelled containing rod shaped ${ }^{60} \mathrm{Co}$ sources, Figure 2. The ICRP gamma dose is obtained in ring-shaped scoring regions around the flask.
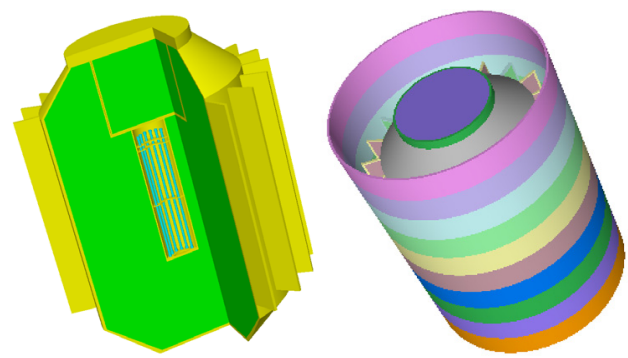

Figure 2. Fuel flask model (left, with cut-out quarter, source in blue) and scoring zones (right).

The results from the FG and the IGES model agree within one standard deviation.

\subsection{Pipeworks}

A system of steel pipes filled with a water/MOX fuel mixture has been modelled, Figure 3, the fluid representing a gamma source. The gamma spectrum is defined in 18 energy groups (from 0.01 to $4 \mathrm{Mev}$ ). The scoring region is a cylinder of water (coloured violet in Figure 3).

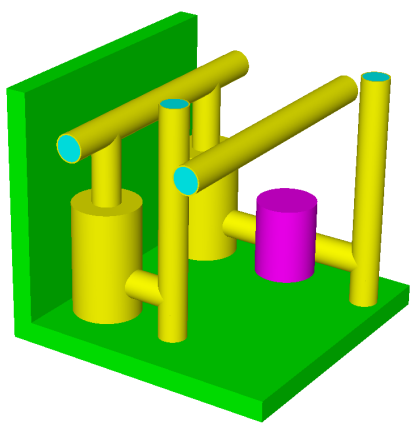

Figure 3. The pipeworks model. The violet body represents the scoring volume. The Neutron source is coloured blue.

The count rates in the scoring volume have been compared in four energy groups and the FG and IGES models were found to agree within one standard deviation. 


\subsection{Hexagonal pipe}

This model consists of 6 steel plates forming a hexagonal pipe. Each plate has several cut-outs of various shapes - this is a 'made up' model to challenge the constructive solid geometry (CSG) conversion of the CAD import. A spherical gamma source (single $1.33 \mathrm{Mev}$ line) is inside the pipe. There are 6 scoring regions at the top (Figure 4).

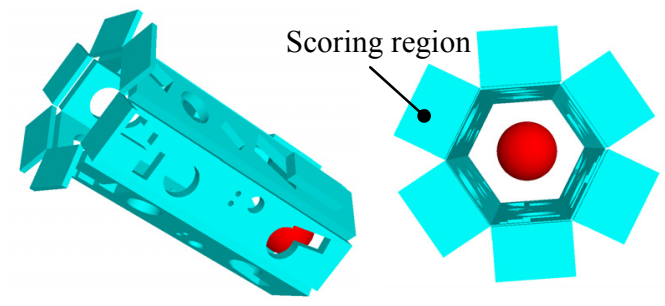

Figure 4. The Hexagonal pipe model (source is marked red).

The fluxes in the energy group around $1.33 \mathrm{MeV}$ for the 6 scoring regions are compared. The results of the FG and the IGES model agree within one standard deviation.

\subsection{TRISO fuel rod}

A part of a fuel rod containing TRISO (Tristructural-isotropic) fuel particles has been modelled, Figure 5. The rod consists of five equal axial sections ( $7 \mathrm{~mm}$ long, 6mm radius) each containing 67 particles in a Graphite matrix. The mantle area of each rod section is covered by a scoring region.
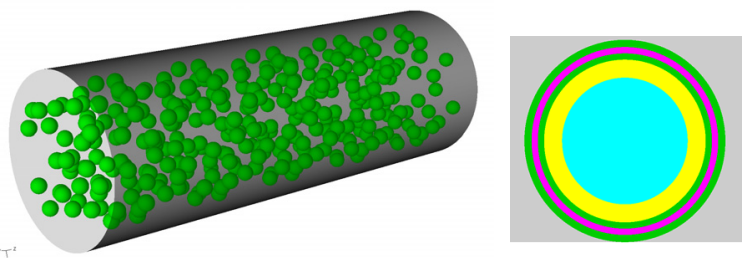

Figure 5. The Triso fuel rod model (left). Layers of one particle (right) from centre outwards: UCO, Graphite, PyC, $\mathrm{SiC}, \mathrm{PyC}$. Radius of particle: $0.56 \mathrm{~mm}$.

For the core of each particle a ${ }^{235} \mathrm{U}$ Neutron spectrum is used. The Neutron fluxes in the 5 scoring regions for the FG and the IGES model agree within one standard deviation.

\subsection{Gamma detector}

Figure 6 shows the setup of two Germanium detectors facing four Gamma sources. The detector models are based on existing devices and have a diameter of $8.3 \mathrm{~cm}$. Each source consists of a rectangular box holding a ${ }^{241} \mathrm{Am}$ bead of $0.5 \mathrm{~mm}$ radius.

The count rates in the detector closest to the surface have been compared in a set of energy groups. The results from the FG and the IGES model agree very well within statistical errors.

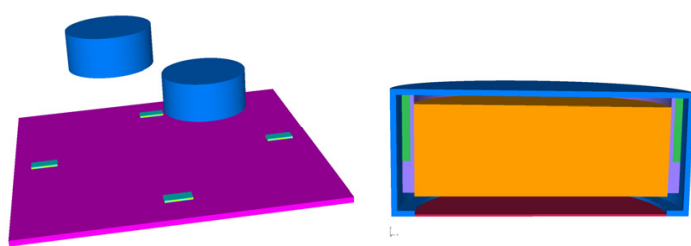

Figure 6. The Gamma detector model (left) and the cross section through one detector (right).

\subsection{Fuel flask}

This flask model comprises a water filled, layered steel/lead container holding 5 boxes with 36 fuel rods (3.4\% enriched Uranium with steel clad) each. The Neutron spectrum is given in 13 energy groups from $0.11-14.6 \mathrm{MeV}$. The scoring regions are a set of 3 concentric rings covering the circular top of the flask.
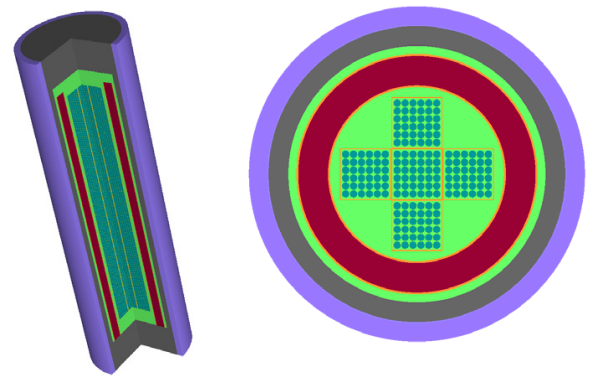

Figure 7. Fuel flask model (water, lead and steel are marked green, red and grey, respectively).

Comparing the Neutron dose in the scoring regions, the FG and the IGES model show agreement within one standard deviation.

\subsection{Human body}

A polygon surface model of a human body has been exported in STL format. A $6 \mathrm{Mev}$ neutron line source was placed above the body and neutron flux scored in 2 energy groups in scoring regions below the body. For the comparison calculation a development version of MCBEND was employed allowing use of a new polygon surface FG body (POLY). The two calculations used different routes through the code for geometry tracking but because the data source for the polygons was the same this example verifies that the STL import behaves the same as the POLY body. As expected there was very close agreement between the calculations.

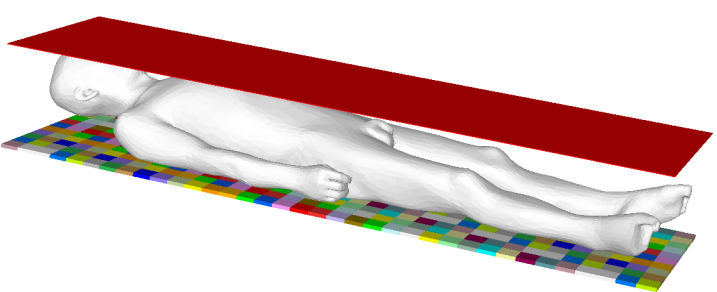

Figure 8. Polygon surface human body (neutron source, red, on top and the scoring mesh shown below). 


\section{Run time comparison}

Reference [2] notes that the tetrahedral mesh provides runtime performance comparable to the FG / Hole geometry, typically ranging from a bit faster than standard MCBEND to a factor of 2 slower.

The ratios of the runtimes for the IGES cases considered above (Table 1) demonstrate that the runtimes can differ significantly - they are strongly dependent on the model and the way the model is represented in the IGES file.

Table 1. Ratio of runtimes of the IGES and FG cases for the models considered.

\begin{tabular}{cc}
\hline Case & Runtime ratio IGES/FG \\
\hline Storage room & 13.0 \\
Transport flask & 84.8 \\
Pipeworks & 100.0 \\
Hexagonal pipe & 3.5 \\
TRISO fuel rod & 57.1 \\
Gamma detector & 4.8 \\
Fuel flask & 282.3 \\
Human Body & $2.4 \quad$ (STL/FG) \\
\hline
\end{tabular}

\section{Discussion}

The simple and seamless integration of CAD geometries in a MCBEND model using the 'IGES body' provides the user with flexibility in setting up models. It removes the need to re-create the geometry for radiation transport calculations when a CAD model already exists. Imported IGES format geometries are not approximated; MCBEND uses exactly the same geometry defined in the CAD software.

The runtimes of models with (IGES) CAD import are longer compared to the equivalent FG model. This runtime difference is strongly dependent on the level of complexity and the combination of materials, but also on the way the IGES model has been set up and the save options used in the CAD software. MCBEND gives accurate and correct results for CAD based models. In the next stage of the MCBEND development the performance of the code when using IGES based models will be addressed.

\section{Conclusion}

MCBEND11A Release Update 0 provides several methods for importing geometry models from CAD programs. These can be easily integrated and used in combination with all existing MCBEND geometry capabilities.

The import of IGES formats provides the greatest flexibility and accurate representation of all surfaces available. Where surfaces can be identified as part of a simple body they are treated as such to increase performance. Run times are model dependent and range from a few times slower to a few hundred times slower than standard MCBEND geometry.

The use of the tetrahedral mesh or polygon surface formats provides a higher performance alternative to the IGES import, at the expense of geometric aproximation of curved surfaces. Run times are comparable and sometimes faster than the equivalent models using standard MCBEND geometry.

The testing indicates that the CAD geometry import is working accurately and effort now focuses on improving the performance of the IGES import.

\section{Acknowledgements}

The authors wish to acknowledge the other MCBEND development team members: Malcolm Armishaw, Geoff Dobson, Simon Richards, Ray Perry, (Amec), Keith Searson and Fabrice Fleurot (Sellafield Ltd).

\section{References}

[1] P. Cowan, G. Dobson and J. Martin, Release of MCBEND 11, Proc. $12^{\text {th }}$ International Conference on Radiation Shielding (ICRS-12) and $17^{\text {th }}$ Topical Meeting on Radiation Protection and Shielding (RPSD-2012), Nara, Japan (September 2012).

[2] T. Barker, A. Bird, R. Thetford and A.J. Cooper, Use of tetrahedral mesh geometry to import a converted CAD file for shielding and criticality calculations with MONK and MCBEND, Proc. 11th International Conference on Radiation Shielding (ICRS-11) and $14^{\text {th }}$ Topical Meeting on Radiation Protection and Shielding (RPS 2008), Georgia, USA (April 2008)

[3] F. Fleurot, K. Searson and A.J. Cooper, OiNC: Criticality benchmark models in CAD: CAD faster than CSG importing CAD data for shielding and criticality calculations with MONK and MCBEND. Proc. International Conference on Nuclear Criticality (ICNC-11), Edinburgh, UK (September 2011).

[4] A. Bird, T. Fry and D. Hanlon, Release of Visual-Workshop2, A model viewer, editor and results display package for the ANSWERS Shielding and Criticality codes. Proc. International Conference on Nuclear Criticality (ICNC-11), Edinburgh, UK (September 2011). 